\title{
Fluidodinâmica Computacional na Simulação Realística de Reatores Nucleares de Leito de Bolas Operando a Potência Reduzida em Condiciones de Perda de Refrigerante
}

\section{Computational Fluid Dynamic in Realistic Simulation of Pebble Bed Nuclear Reactors Operating at Reduced Power in Loss of Coolant Conditions}

Uebert G. Moreira ${ }^{1}$, Dany S. Dominguez², Leorlen Y. R. Mazaira ${ }^{3}$, Carlos R. G. Hernández ${ }^{4}$, Carlos A. B. de Oliveira Lira ${ }^{5}$

\section{RESUMO}

A viabilidade tecnológica de sistemas nucleares depende de um sistema de segurança eficiente na operação de suas plantas. Para os promissores reatores de temperatura muito elevada refrigerados a gás com núcleo de leito de bolas, esforços estão sendo empreendidos com alguns reatores experimentais, a exemplo do modelo HTR (High Temperature Reactor)-10. Neste trabalho, são realizadas simulações termohidráulicas do núcleo do reator de tipo leito de bolas HTR-10, usado como estudo de caso, para investigar, dentre outros fatores, o efeito da redução da taxa de escoamento do fluido refrigerante no perfil de temperatura do núcleo. Adotou-se técnicas de dinâmica de fluidos computacional (CFD) com uma abordagem realística para modelar e simular uma geometria que representa uma coluna da seção central do núcleo do reator. Para simplificar a disposição dos elementos combustíveis no interior do núcleo, é usado o arranjo cúbico centrado no corpo (BCC). Os dados de entrada usados foram extraídos do relatório técnico de problemas de benchmark divulgado pela IAEA (TECDOC-1694). Os resultados mostram os perfis de temperatura no interior das bolas de combustível considerando regimes de perda de refrigerante. As temperaturas máximas alcançadas não excedem o limite de segurança projetado para o tipo de combustível nuclear do reator.

Palavras-chave: Simulação CFD. Leito de Bolas. Reator Nuclear. Perda de Refrigerante.
${ }^{1}$ Mestre em Modelagem Computacional. Universidade Federal do Oeste Baiano.

E-mail:

uebert.gmoreira@gmail.com

${ }^{2}$ Doutor em Modelagem

Computacional. Universidade

Estadual de Santa Cruz.

${ }^{3}$ Doutor em Tecnologias Energéticas e Nucleares. Universidade Federal de Pernambuco.

${ }^{4}$ Doutor em Ciências Técnicas. Instituto Superior de Tecnologías y Ciencias Aplicadas.

${ }^{5}$ Doutor em Engenharia Nuclear. Universidade Federal de Pernambuco.

\section{ABSTRACT}

The technological feasibility of nuclear systems depends on an efficient safety system in the operation of your plants. For promising very high temperature gas cooled reactors (VHTRs), efforts are being made with some experimental reactors, such as the HTR (High Temperature Reactors)-10 model. In this work, thermohydraulic simulations of the core of the HTR-10 pebble bed reactor, used as a case study, are carried out to investigate, among other factors, the effect of reducing the flow rate of the coolant in the core temperature profile. Computational fluid dynamics (CFD) techniques with a realistic approach were adopted to model and simulate a geometry representing the central section column of the reactor core. To simplify the arrangement of the fuel elements inside the core, was used the body centered cubic (BCC) arrangement. The input data used were taken technical report on benchmark problems from by IAEA (TECDOC1694). The results show the temperature profiles inside the fuel balls considering coolant loss regimes. The maximum temperatures reached do not exceed the designed safety limit for the nuclear fuel type of the reactor.

Keywords: CFD Simulation. Pebble Bed. Nuclear Reactor. Coolant Loss. 


\section{INTRODUCAAO}

Estudos de desenvolvimento socioeconômico projetam aumentos contínuos e significativos da demanda energética mundial nas próximas décadas. Este cenário apresenta como pano de fundo as preocupações ambientais com atividades que promovem o agravamento do efeito estufa, em particular, a emissão de dióxido de carbono pela queima de combustíveis fósseis. Diante disso, a energia nuclear se credencia por apresentar, dentre outras vantagens, alta densidade energética com baixo impacto ambiental. Entretanto, a expansão da indústria nuclear depende do enfrentamento de várias questões apresentadas pela (IAEA, 2013), entre as quais se inclui o melhoramento dos níveis de segurança das centrais nucleares.

Tecnologias de energia nuclear mais inovadoras, conhecidas como sistemas nucleares de Geração IV, estão sendo desenvolvidas através de fóruns de cooperação internacional. Entre os reatores dessa nova geração, destacam-se os reatores de temperatura muito elevada refrigerados a gás (VHTRs, Very High Temperature Reactors) pela maturidade conceitual e possibilidade de outras aplicações. A finalidade destes reatores inclui, além da produção de energia elétrica, processos de geração de calor para aplicações industriais tais como para refinarias, petroquímica, metalurgia, produção de hidrogênio, de combustíveis sintéticos do carvão, biomassa ou captura de $\mathrm{CO}_{2}$ (FUTTERER et. al., 2014). Segurança inerente, baixa densidade de potência e alta eficiência de conversão são outros importantes atrativos deste reator.

Os VHTRs utilizam partículas de combustível TRISO (Tristructural Isotropic) constituídas por um núcleo físsil revestido por camadas refratárias de grafite responsáveis pela retenção dos produtos de fissão a elevadas temperaturas. Em um conceito promissor estudado para o núcleo do reator, as partículas TRISO são inseridas em matrizes de grafite em formato esférico distribuídas aleatoriamente no espaço destinado ao núcleo do reator, resultando no leito de bolas com formato anular ou cilíndrico cercado por blocos refletores de grafite.

Como parte de um Programa de Pesquisa Coordenado da IAEA (International Atomic Energy Agency) sobre a tecnologia combustível do reator VHTR, um conjunto de atividades de benchmark foi desenvolvido para comparar códigos para performance de combustível sob operação normal e transientes operacionais (IAEA, 2013). Dentre as questões apresentadas, o segundo problema de benchmark sugere investigar os impactos da perda de escoamento de hélio para o circuito primário sem scram (desligamento automático do 
reator). Na ocorrência de um acidente deste tipo, a temperatura dos elementos combustíveis poderia aumentar descontroladamente até níveis que ocasionem a liberação dos produtos de fissão.

Os sistemas de controle, de proteção e o comportamento dos componentes envolvidos devem ser testados e verificados, em condições normais e adversas, para garantir a segurança e a integridade do núcleo do reator. Nesta perspectiva, a termo hidráulica possibilita investigar se as grandezas operacionais como temperatura e pressão permanecem dentro das margens de segurança projetadas, incluindo as temperaturas atingidas pelo combustível.

Neste trabalho, executam-se simulações termo hidráulicas estacionárias do núcleo de reatores de temperatura muito elevada do tipo leito de bolas para estudo do impacto da perda da taxa de escoamento de fluido refrigerante em relação ao perfil de temperatura do núcleo. A mudança de reatividade, bem como outros mecanismos de segurança do reator, e o ingresso (ou entrada) de ar, foram desconsiderados. Selecionado pela (IAEA, 2013) como uma das referências para avaliação do desempenho termo hidráulico e comparações experimentais associadas a reatores de alta temperatura refrigerados a gás, o reator de teste de leito de bolas HTR (High Temperature Reactor)-10 é utilizado como estudo de caso neste trabalho.

Diante dos padrões complexos de fluxo no interior do leito de bolas, os cálculos são executados com o uso de técnicas de dinâmica de fluidos computacional (CFD). Para este tipo de simulação, adotou-se uma abordagem realística usando o código ANSYS CFX, versão 14.0. Resultados obtidos por (WU et. al., 2010) indicam que as características termo hidráulicas anisotrópicas no interior de leitos de bolas empacotados podem ser simuladas de forma mais apropriada com essa abordagem. Como configuração geométrica selecionou-se o arranjo BCC (Body Centered Cubic) indicada pelos trabalhos de (SILVA, et. al., 2016) e (FERNG e LIN, 2013) como a configuração que obtém temperaturas mais altas no interior do núcleo, se comparada a outros arranjos de bolas fixadas. Diante das limitações computacionais, optou-se por utilizar um modelo geométrico que representa uma coluna da seção central do núcleo do reator, pois é na região central do núcleo onde os elementos combustíveis alcançam maior temperatura.

Dentre as pesquisas recentes para investigar ou predizer comportamentos dos elementos do núcleo do HTR-10 em condições de operação normal e de acidentes, os resultados apresentados em (SILVA, et. al., 2016) e (MOREIRA, et. al., 2017) descreveram 
os parâmetros termo hidráulicos do núcleo do reator HTR-10 usando uma metodologia CFD com abordagem realista; no trabalho de (FERNG e CHI, 2012) investigou-se o acidente de entrada de ar no reator para obter características termo hidráulicas e verificar o índice de corrosão do grafite por oxidação; outro teste para demonstração de segurança, se tratando de um transiente antecipado sem desligamento automático e com inserção de reatividade, foi simulado por (CHEN, DONG e ZHANG, 2016); em (SCARI, et. al., 2016), os autores apresentaram um modelo térmico para simular o comportamento estacionário e transitório do reator; modelos de turbulência na temperatura e na velocidade do refrigerante no núcleo do reator, por sua vez, são analisados por (SUBETKI, 2016).

A seção seguinte discutirá as características do modelo computacional utilizado neste trabalho, incluindo domínio geométrico, esquema de discretização, modelos matemáticos envolvidos na simulação e condições de contorno. Os resultados e discussões são abordados na seção 3 . Por fim, na seção 4 , são oferecidos os comentários finais e sugestões para trabalhos futuros.

\section{DOMINIO COMPUTACIONAL E CONDICCOES DE CONTORNO}

O HTR-10 é um reator de espectro de nêutrons térmico, moderado a grafite e refrigerado por gás hélio. Características físicas e operacionais deste reator estão disponíveis em (IAEA, 2013). O núcleo do HTR-10, designado para uma potência térmica de $10^{7} \mathrm{~W}$, é revestido por refletores de grafite e constituído por um leito de bolas com diâmetro de 1,8 $\mathrm{m}$ e altura média de 1,97 $\mathrm{m}$. Cada esfera de combustível é composta por uma região interna de $5 \times 10^{-2} \mathrm{~m}$ de diâmetro (onde as partículas TRISO são inseridas) revestida por uma camada de grafite sem combustível com $5 \times 10^{-3} \mathrm{~m}$ de espessura. $\mathrm{O}$ revestimento das partículas TRISO, por sua vez, compreende três camadas de carbono pirolítico e uma camada de carbono silício, responsáveis por reter os produtos decorrentes da fissão até uma temperatura de 1873 K (KOK, 2013), sob pena de colapso de sua estrutura.

As passagens do refrigerante no interior do vaso de pressão são ilustradas pela Figura 1. O gás frio é bombeado para o circuito primário e escoa através do espaço intersticial dentro da barreira de contimento do núcleo. O refrigerante flui de cima para baixo através do leito de bolas do núcleo, sendo aquecido pelos elementos combustíveis nesse processo; em seguida, o hélio quente sai do núcleo do reator através de um duto na direção do 
gerador de vapor, onde é esfriado e novamente bombeado para o núcleo do reator pelo circulador de hélio.
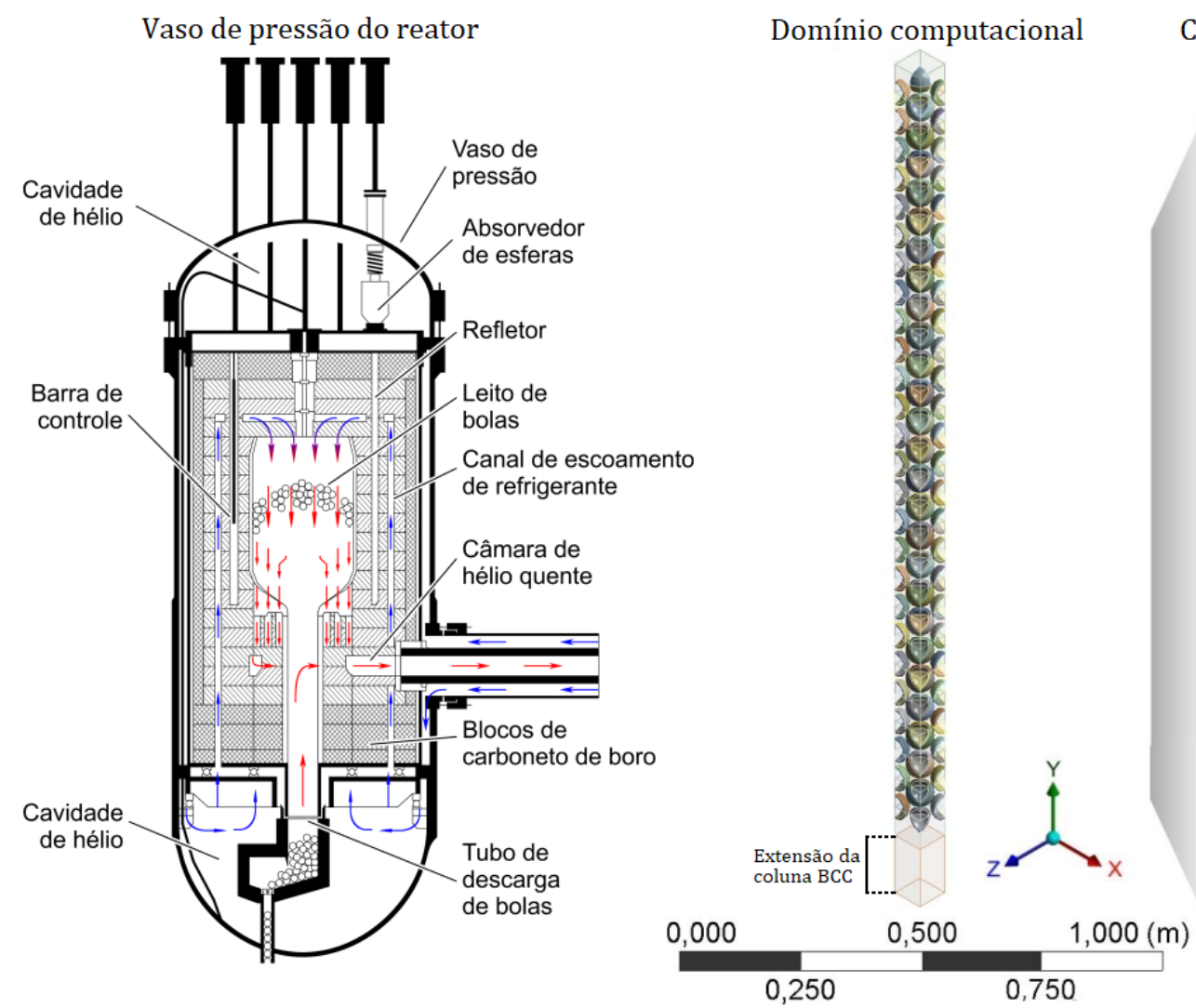

Camadas de potência

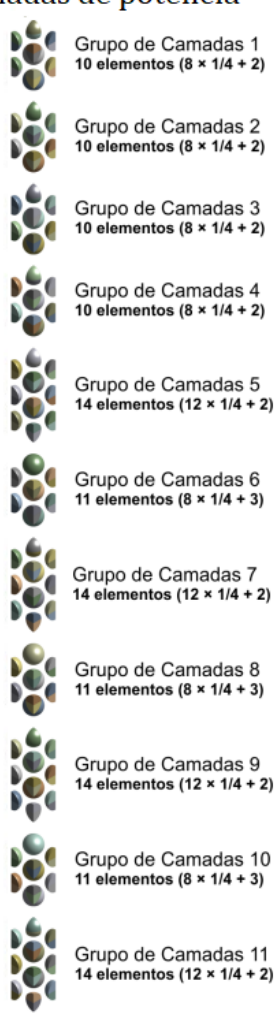

Figura 1. Passagens de escoamento do refrigerante no interior do vaso de pressão (à esquerda) e domínio computacional da coluna central do núcleo do HTR-10 com distribuição de camadas de potência (à direita)

Em função do alto custo computacional requerido para simulação do núcleo completo do reator HTR-10, optou-se por uma geometria que representa uma coluna da seção central do núcleo com altura de 1,94 m. A geometria proposta, ilustrado na Figura 1, foi construída pela replicação, na direção vertical, de 27 arranjos cúbicos $\mathrm{BCC}$, dispondo de 25 bolas de combustível completas e 52 quartos de bolas, distribuídas em 11 grupos de potências (IAEA, 2013). Na parte inferior da coluna, uma extensão de 0,1438 m foi adicionada para garantir que as condições de desenvolvimento do escoamento do fluido sejam plenamente alcançadas.

Para melhor descrição do fenômeno convectivo, na etapa de discretização da geometria pela aplicação do método dos volumes finitos (VERSTEEG e MALALASEKERA, 2007), malhas mais refinadas foram aplicadas (10 camadas definidas) nas interfaces entre regiões de domínio do refrigerante e o revestimento externo do grafite, conforme ilustra 
Figura 2; a espessura de cada camada aumenta a uma razão de $20 \%$ à medida em que se distancia das regiões de interface e a soma das espessuras de todas as camadas totalizam $7 \times 10^{-4} \mathrm{~m}$. A malha gerada possui 12.554 .181 nodes e 39.491 .159 elementos. Para garantir independência dos resultados em relação à configuração da malha, resultados extraídos por simulações com 0 uso de quatro malhas distintas foram comparados, com a obtenção de desvios relativos nas maiores temperaturas inferiores a $1 \%$.

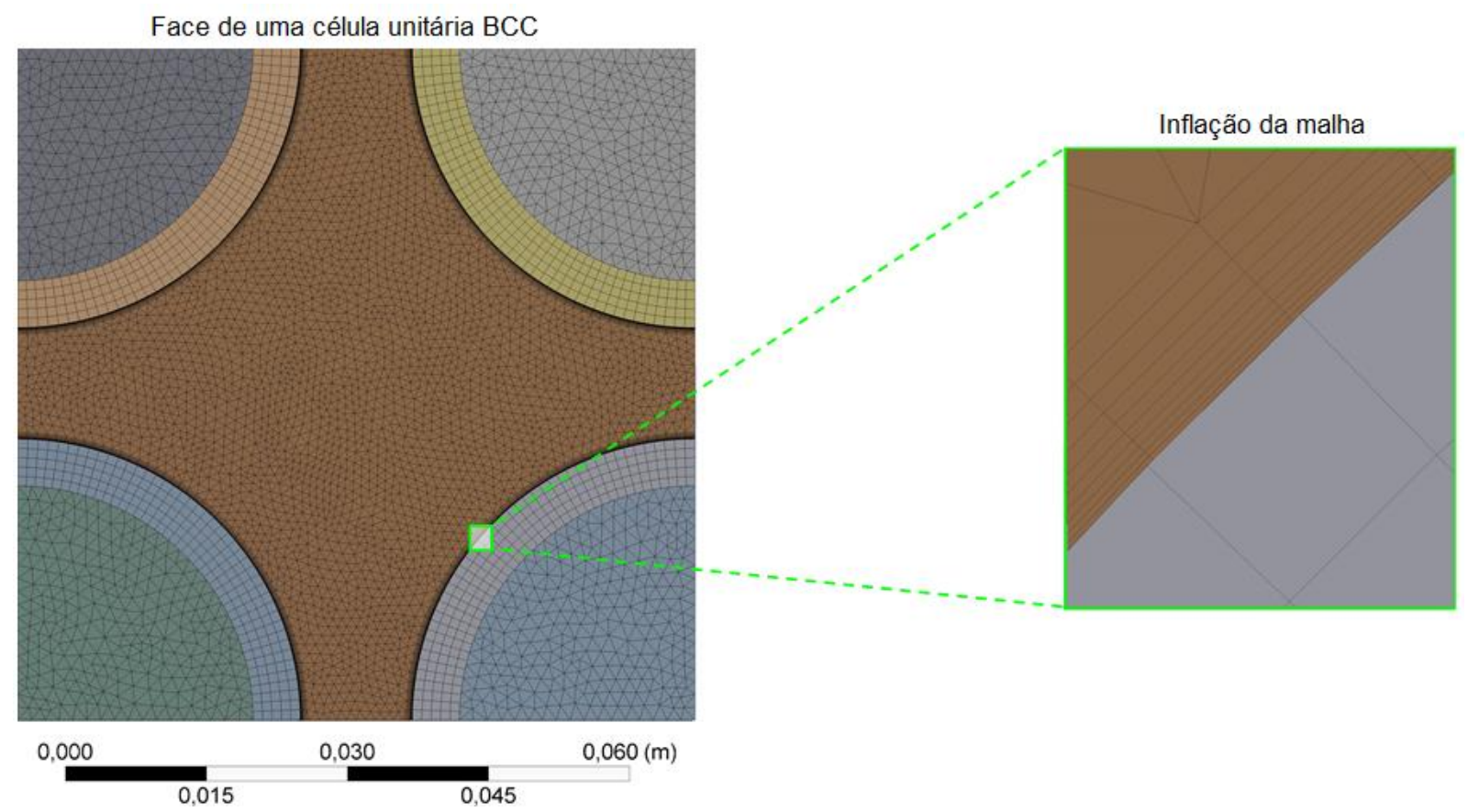

Figura 2. Inflação de malha da face de uma célula unitária BCC

Para simulação das características termo hidráulicas do núcleo do HTR-10, equações diferenciais parciais conhecidas como equações de governança são usadas para caracterização física do escoamento do fluido (TU, YEOH e LIU, 2013). Os termos dependentes da variável tempo, são considerados nulos neste trabalho. O modelo matemático inclui, também, o modelo de turbulência $k-\varepsilon$ padrão e considera a compressibilidade do hélio. Em relação às propriedades físicas dos materiais (hélio, grafite e combustível), seguiram-se recomendações presentes no benchmark da (IAEA, 2013) e no manual de normas da comissão alemã de segurança nuclear para reatores de leito de bolas (KTA, 1978).

Sobre as condições de contorno, são consideradas, no presente trabalho, para as regiões de entrada e saída, simetria e de parede. Para o regime escoamento é assumida 
velocidade subsônica com direção normal ao plano de entrada. Diante do desconhecimento da intensidade de turbulência, adotou-se a definição de 5\% de intensidade e taxa de viscosidade equivalente 10, conforme recomendação de (ANSYS, 2011). Na região de saída, o perfil de pressão escolhido é associado a uma pressão média definida estaticamente em $0 \mathrm{~Pa}$.

A situação apresentada no segundo problema de benchmark da (IAEA, 2013), estabelece que o reator atingiu operação em estado estacionário a uma carga parcial correspondente a $30 \%$ da potência térmica total, isto é, $3 \times 10^{6} \mathrm{~W}$; a pressão primária de hélio na operação de carga parcial em estado estacionário é de $2,5 \times 10^{6} \mathrm{~Pa}$; as temperaturas médias do hélio na entrada e na saída são de, respectivamente, $523 \mathrm{~K} \mathrm{e} 923 \mathrm{~K}$. A taxa de escoamento de hélio para o núcleo, por sua vez, é definida pelas diferenças entre temperatura, pressão do hélio e considerando, também a redução de potência. A equação clássica para este cálculo analítico de balanço energético, Equação 1, compara a variação de temperatura $\Delta T$, durante o aquecimento $Q$ do núcleo, com certa taxa de fluxo do hélio, $m_{v a z a ̃ o}$, que possui capacidade de calor específico $C_{p}$.

$$
Q=m_{\text {vazão }} C_{p} \Delta T
$$

Pela aplicação da Equação 1, obtém-se uma taxa de escoamento de hélio para o núcleo correspondente a $1,44 \mathrm{Kg} / \mathrm{s}$. Considerando o percentual de hélio que efetivamente refrigera os elementos combustíveis (estimado em $87 \%$ ), e a razão entre a área da seção de entrada do refrigerante no núcleo e a área da seção de entrada na coluna de $\mathrm{BCC}$, a taxa de fluxo de massa na entrada do domínio foi definida em 2,545× $10^{-3} \mathrm{Kg} / \mathrm{s}$.

A investigação do comportamento do núcleo quando há perda de escoamento de hélio para o circuito primário sem scram é iniciada, no instante zero, com o desligamento do ventilador primário de hélio, provocando redução do circulador de hélio para aproximadamente zero em 10 segundos. Supondo uma redução linear da densidade de hélio no núcleo do reator em relação ao tempo, obtém-se que a cada segundo o núcleo perde $10 \%$ da taxa de refrigerante definida. Diante disso, para as simulações deste trabalho, foram consideradas reduções da vazão mássica de refrigerante com uma razão de $10 \%$ em relação à quantidade definida.

A potência térmica gerada no núcleo constitui outra condição crítica de entrada para as simulações no presente trabalho. Os valores admitidos para a potência do núcleo, foram extraídos do resultado experimental do teste de perda de escoamento de refrigerante para o núcleo do HTR-10 sem scram fornecido pela (IAEA, 2013). Como o presente trabalho se 
restringe a simulações termo hidráulicas estacionárias, o comportamento transiente dos coeficientes de reatividade é desconsiderado.

A turbulência do fluido no leito de bolas é simulada com a resolução das equações de RANS (Reynolds-Averaged Navier Stokes) através da introdução do modelo de turbulência k- $\varepsilon$ padrão. Para tratamento numérico de turbulência utilizou-se o esquema de advecção High Resolution de Primeira Ordem. Como critério de convergência para as equações de governança estabeleceu-se que o valor da norma RMS (Root Mean Square) dos resíduos fosse inferior a $10^{-4}$.

\section{RESULTADOS E DISCUSSOES}

Para atender ao estudo proposto, considerou-se reduções da vazão mássica de refrigerante a uma razão de $10 \%$ em relação à quantidade definida. Os valores obtidos para a taxa de escoamento foram usados em simulações sequenciais distintas. A redução da taxa de escoamento ocorreu até que a temperatura máxima alcançada pelos elementos combustíveis esteja próxima do limite de segurança recomendado. As simulações convergiram para fluxos de refrigerante correspondentes à 100\%, 90\%, 80\%, 70\%, 60\%, $50 \%$ e $45 \%$ da taxa de escoamento definida. Não se alcançou o nível de convergência estipulado reduzindo o fluxo de refrigerante a 40\%; embora o resultado de tal simulação tenha registrado $1.871 \mathrm{~K}$, a propagação dos resíduos apresentou alto grau de oscilação ao longo de 1.000 iterações computadas. A instabilidade dos resíduos destas simulações pode estar associada ao fato do fenômeno de perda de refrigerante ser intrinsecamente transiente; neste caso, a simulação estacionária pode apresentar problemas de convergência, estes que, normalmente, se manifestam mais intensamente em situações com menor fluxo de refrigerante.

Na Figura 3 são mostrados os perfis de temperaturas no plano axial central da coluna BCC do leito de bolas para os diferentes fluxos mássicos de refrigerante. Os resultados apontam para o esperado aumento gradativo da temperatura ao reduzir o fluxo de hélio mantendo-se os demais parâmetros e ao descermos pela coluna na direção axial até que se alcance um valor máximo. A maior temperatura obtida, com $45 \%$ da vazão mássica de refrigerante, está pouco menos de $200 \mathrm{~K}$ abaixo do limite de segurança. Diante dessa amplitude, percebe-se que ainda há margem para redução do fluxo de refrigerante sem que a temperatura do combustível transcenda o nível de segurança do projeto. 
MOREIRA, U. G.; DOMINGUEZ, D. S.; MAZAIRA, L. Y. R.; HERNANDEZ, C. R. G.; OLIVEIRA LIRA, C. A. B.

Fluidodinâmica Computacional na Simulação Realística de Reatores Nucleares de Leito de Bolas Operando a Potência Reduzida em Condiciones de Perda de Refrigerante.

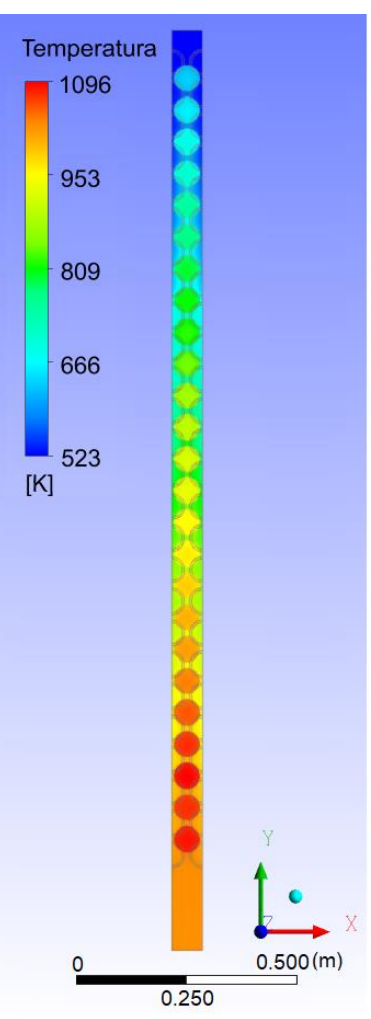

(a)

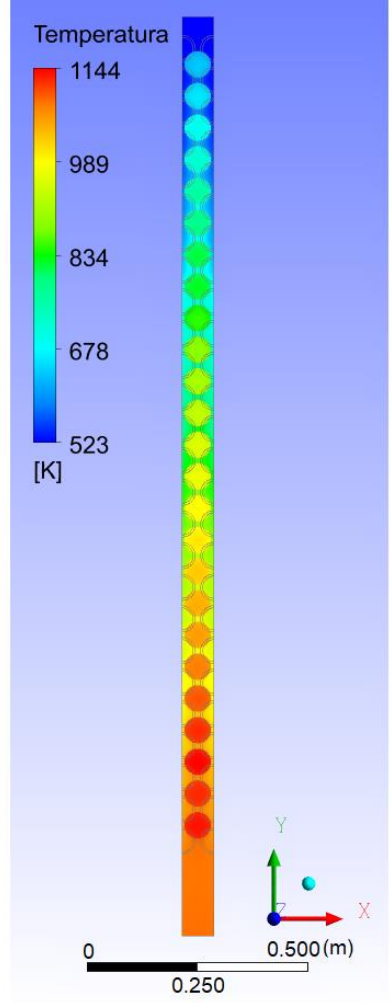

(b)

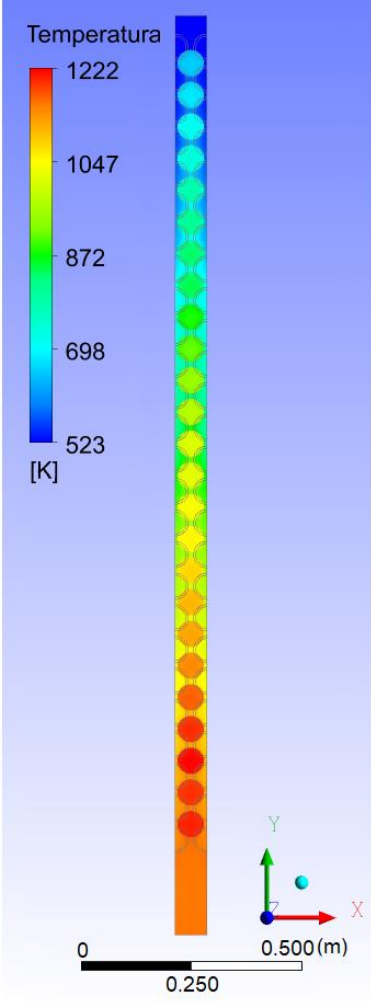

(c)

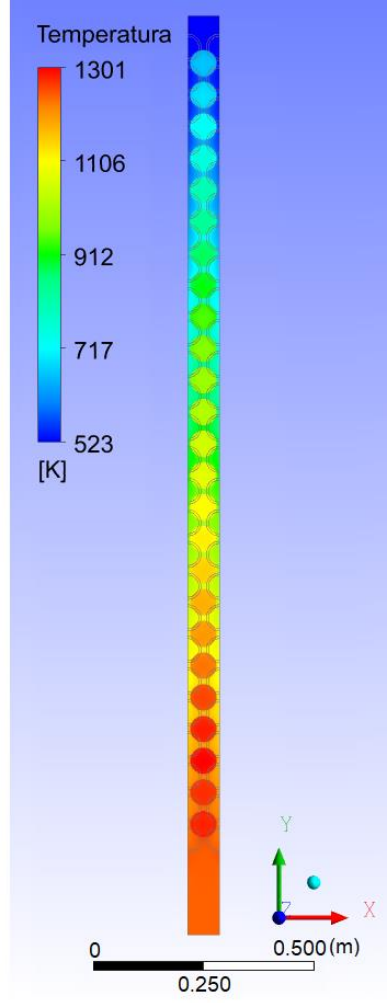

(d)

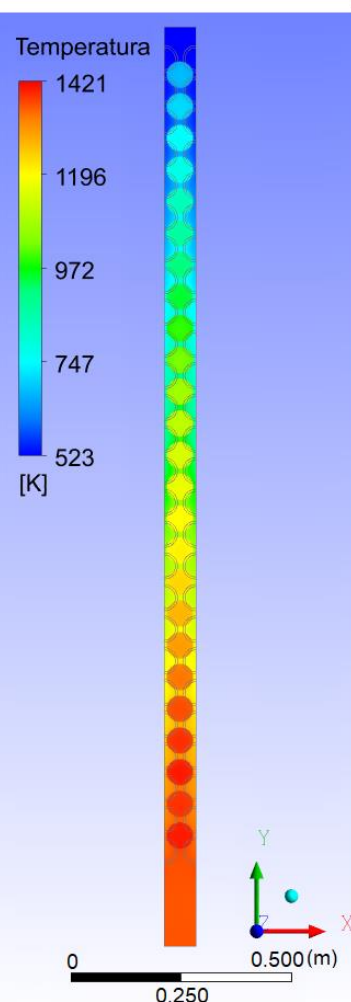

(e)

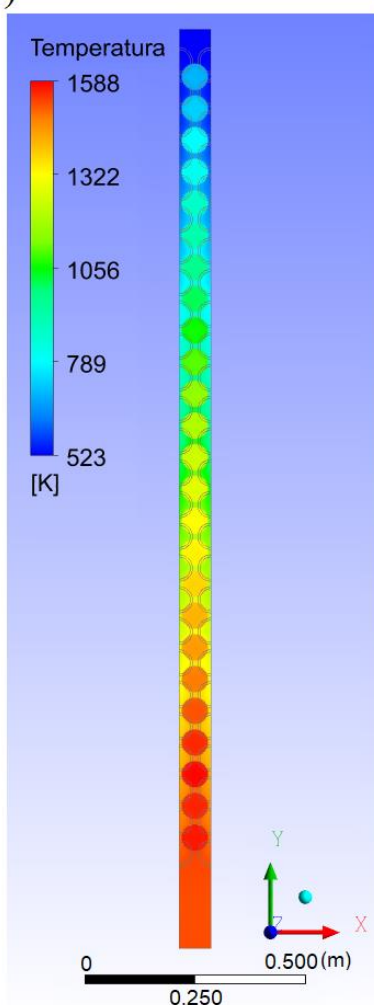

(f)

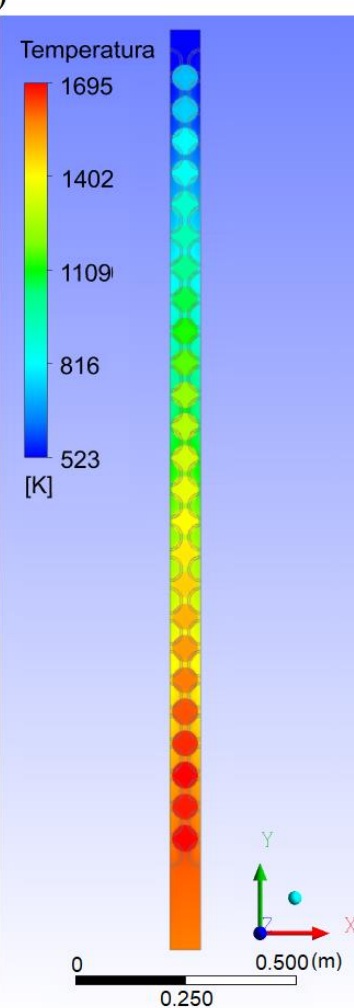

(g)

Figura 3. Distribuição axial de temperaturas na coluna BCC da região central do núcleo do HTR-10 com carga parcial de $30 \%$ da potência para os casos de $100 \%$ (a), $90 \%$ (b), $80 \%$ (c), $70 \%$ (d), $60 \%$ (e), $50 \%$ (f) e $45 \%$ (g) da quantidade de refrigerante definida 
Em relação à evolução axial da temperatura do combustível, conforme ilustra a Figura 4, as maiores temperaturas são registradas em profundidade de 1,69 m, após a qual se assiste um decaimento da temperatura devido ao fato da seção inferior do leito de bolas gerar menos potência que os demais grupos. Percebe-se também que o decaimento de temperatura apresenta pouca profundidade, permitindo que o crescimento que o segue, fique bem próximo do pico de temperatura. A temperatura alcançada na medida em que o núcleo perde refrigerante é resumida na Figura 4. Supondo que o aumento da temperatura apresente comportamento próximo ao do polinômio de terceiro grau que interpola os sete pontos do gráfico, o fluxo de refrigerante no núcleo poderia ser reduzido até que restasse um percentual correspondente a $39 \%$.
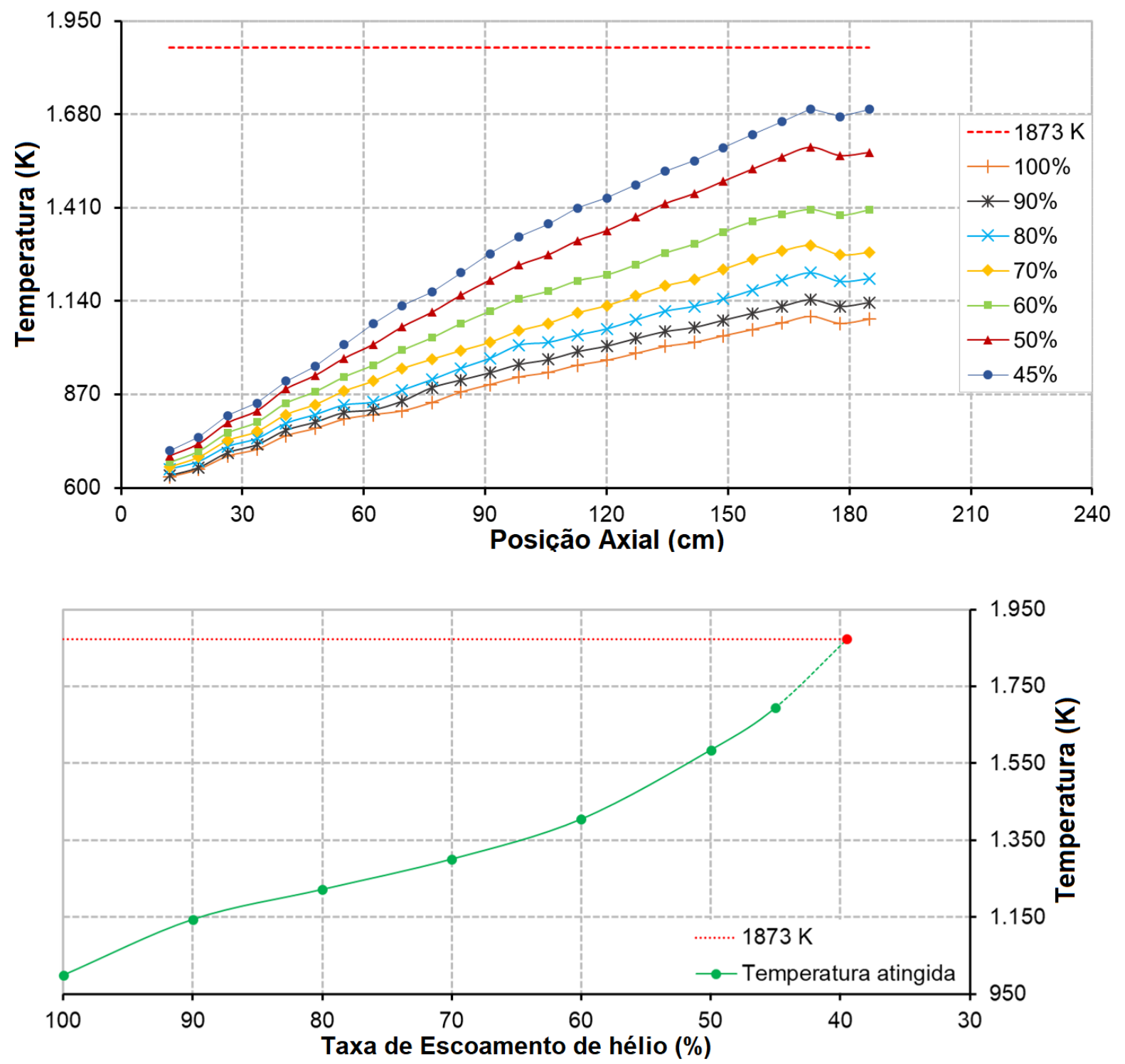

Figura 4. Temperatura do combustível em relação à posição axial no eixo central (acima); temperaturas máximas em relação à taxa de escoamento de hélio para a coluna BCC central do núcleo (abaixo) 
De acordo com os dados apresentados na Figura 3 e na Figura 4 é possível concluir que há certa regularidade nos resultados das simulações realizadas em relação à evolução da temperatura ao longo do plano central da coluna, bem como nas temperaturas máximas dos diversos componentes do leito de bolas (hélio, camada de grafite e elemento combustível). Observa-se, por exemplo, que as curvas das temperaturas na direção axial, para os casos de fluxo de refrigerante estudados, apresentam intervalos de crescimento e decrescimento semelhantes.

Ao se deter na análise da temperatura média de saída do gás hélio, ilustrada na Figura 5, para o fluxo de refrigerante definido, esta apresenta distribuição homogênea aproximadamente $100 \mathrm{~K}$ acima do valor esperado, comportamento semelhante ao identificado pelas simulações do núcleo em plena potência realizadas por (SILVA, et. al., 2016) e com resultados do primeiro problema de benchmark divulgados em (IAEA, 2013).

Por fim, a Figura 5 ilustra a distribuição de temperaturas nas superfícies que contornam a região de combustível da bola que atingiu maior temperatura considerando a quantidade total de escoamento de hélio definida. É possível perceber que temperaturas menores estão registradas na região da esfera disposta de encontro ao fluxo de escoamento axial, havendo maior transferência de calor nesse local; no polo oposto, se concentram as maiores temperaturas na superfície da esfera, sendo, portanto, a região que efetiva menor transferência de calor.
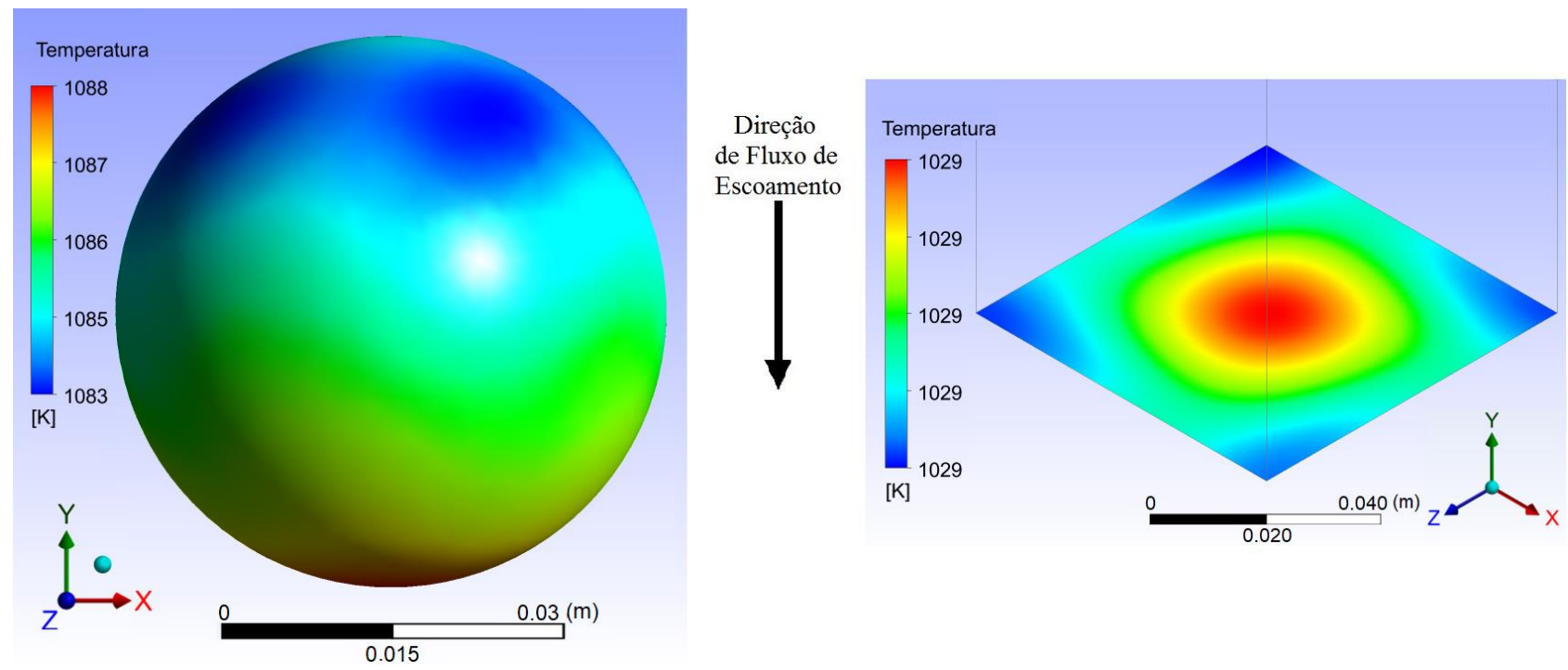

Figura 5. Distribuição de temperatura na superfície da bola da coluna BCC na qual se registrou maior temperatura (à esquerda) e distribuição de temperatura do hélio na região de saída da coluna (à direita) considerando a quantidade de refrigerante total definida 
Diante da ausência de valores de referência para os resultados apresentados nesta seção, a consistência dos resultados obtidos se baseia na coerência do comportamento fenomenológico identificado, e nas concordâncias no que tange à evolução da temperatura dos elementos dos subdomínios ao longo da coluna em comparação com os resultados relacionados ao estado de plena potência do núcleo disponíveis na literatura, como, nos trabalhos de (SILVA, et. al., 2016), (MOREIRA, et. al., 2017) e (IAEA, 2013).

\section{CONCLUÇOES E TRABALHOS FUTUROS}

Para atender aos requisitos de segurança, o reator deve remover passivamente o calor produzido pelo núcleo em várias condições de acidente, mantendo a temperatura máxima do combustível abaixo de $1.873 \mathrm{~K}$ para conter todos os produtos de fissão dentro das camadas de combustível revestido TRISO. O acidente de perda de hélio foi simulado para verificar a temperatura alcançada pelos elementos combustíveis da coluna central do núcleo do HTR-10. Para considerar o aspecto transitório foram consideradas reduções do fluxo de massa do refrigerante.

De acordo com os perfis da distribuição de temperaturas da seção central do domínio computacional, o combustível atinge temperatura máxima de $1.695 \mathrm{~K}$ com 45\% da vazão mássica do refrigerante para a coluna BCC. Observe que desconsiderando a variação de reatividade ou outro mecanismo de segurança, o núcleo do HTR-10 suporta redução significativa do fluxo de massa de hélio no núcleo do reator sem atingir valores de temperatura que prejudiquem a integridade das partículas de TRISO.

Em relação aos trabalhos futuros, pode-se sugerir aqui a simulação transiente do segundo problema de benchmark publicado em (IAEA, 2013). Para simplificação da geometria e do acoplamento neutrônico, sugere-se, também, o uso da geometria BCC usada neste trabalho e das equações de cinética pontual, respectivamente

Para auxiliar na organização da redação da discussão, procure utilizar a mesma ordem realizada na apresentação dos resultados. A discussão deve apresentar a explicação para os resultados verificados em seu estudo. Isso deve ser realizado com base nas premissas estabelecidas. 


\section{AGRADECIMENTOS}

Os autores agradecem à CAPES, FAPESB e FACEPE pelo suporte financeiro provido e ao NBCGIB-UESC (Núcleo de Biologia Computacional e Gestão de Informações Biotecnológicas da Universidade Estadual de Santa Cruz) pela infraestrutura oferecida para o desenvolvimento desta pesquisa

\section{REFERÉNCIAS}

ANSYS. CFX R14 User's Guide. Release 14.0, Help System, Canonsburg, 2011.

CHEN, F.; DONG, Y. e ZHANG, Z. Post-test simulation of the HTR-10 reactivity insertion without scram. Annals of Nuclear Energy, v. 92, 2016, pp. 36-45.

FERNG, Y. M. e CHI, C. W. CFD investigating the air ingress accident for a HTGR simulation of graphite corrosion oxidation. Nuclear Engineering and Design, v. 248, 2012, pp. 55-65.

FERNG, Y. M. e LIN, K. Y. Investigating effects of BCC and FCC arrangements on flow and heat transfer characteristics in pebbles through CFD methodology. Nuclear Engineering and Design, v. 258, 2013, pp. 66-75.

FUTTERER, M. A.; FU, L.; SINK, C.; GROOT, S.; POUCHON, M.; KIM, Y. W.; CARRÉ, F. e TACHIBANA, Y. Status of the very high temperature reactor system. Progress in Nuclear Energy. v. 77, 2014, pp. 266-281.

IAEA. Evaluation of High Temperature Gas Cooled Reac-tor Performance: Benchmark Analysis Related to the PBMR-400, PBMM, GT-MHR, HTR-10 and the ASTRA Critical Facility. International Atomic Energy Agency, Viena, 2013.

KOK, K. D. Nuclear Engineering Handbook. Boca Raton, CRC Press, 2009.

KTA. Reactor Core Design for High-Temperature Gas-Cooled Reactor (Part 1: Calculation of the Material Properties of Helium). Kerntechnischen useshusses (Nuclear Safety Standards Commission), Salzgitter, 1978.

MOREIRA, U. G.; DOMINGUEZ, D. S.; MAZAIRA, L. Y. R.; HERNÁNDEZ, C. R. G.; e LIRA, C. A. B. O. Thermohydraulic Modeling of Very High Temperature Reactors in Regimes with Loss of Coolant using CFD. In: International Nuclear Atlantic Conference - INAC, 2017.

SCARI, M. E.; COSTA, A. L.; PEREIRA, C.; VELASQUEZ, C. E. e VELOSO, M. A. F. Steady state and transient thermal analyses. International Journal of Hydrogen Energy. v. 41, 2016, pp. 7192-7196.

SILVA. A. S.; MAZAIRA, L. Y. R.; DOMINGUEZ, D. S.; HERNÁNDEZ, C. R. G.; e LIRA, C. A. B. O. Uso de Arranjos Cúbicos na Simulação Realística CFD do Núcleo do Reator Nuclear HTR-10. In: Encontro Nacional de Modelagem Computacional - ENMC, 2016.

SUBETKI, M. The effect of turbulence models on coolant temperature and velocity for 
the pebble-bed typed high temperature reactor. In: International Conference on Nuclear Energy Technologies and Sciences, 2015.

TU, J.; YEOH, G. H. e LIU, C. Computational Fluid Dynamics: A Practical Approach. Oxford, Butterworth-Heinemann, 2013.

VERSTEEG, H. K. e MALALASEKERA, W. An Introduction to Computational Fluid Dynamics: The Finite Volume Method. Harlow, Pearson Education, 2007.

WU, C. Y.; FERNG, Y. M.; CHIENF, C. C.; LIU, C. C. Investigating the advantages and disadvantages of realistic approach and porous approach for closely packed pebbles in CFD simulation. Nuclear Engineering and Design. v. 240, 2010, pp. 1151-1159. 